\title{
El universo emocional en las interacciones parentofiliales: un acercamiento al estado del arte
}

\author{
Sandra Liliana Aya-Angarita, Mg. \\ Universidad Santo Tomás, Colombia* \\ Carlos Iván García-Suárez, Ph.D. \\ Universidad de Manizales-Cinde, Colombia**
}

sandraaya@usantotomas.edu.co

\section{Resumen (analítico)}

En el presente artículo de revisión, los autores se proponen develar las maneras en que diversos desarrollos teóricos e investigativos han comprendido el engranaje emocional en las relaciones parentofiliales. Para tal fin, a través de un ejercicio hermenéutico, se analizaron de manera exhaustiva 69 textos publicados en Iberoamérica y el Caribe y algunos países europeos en los últimos 13 años. Tal desarrollo permitió identificar que las emociones en las interacciones parentofiliales se comprenden como mecanismos de control, como hilos entretejidos a lo largo de la vida y como constructos sociohistóricos y culturales. Los hallazgos sugieren además que, pese a que los investigadores del tema reconocen variados métodos e integran diversas perspectivas, no hay claridad sobre las complejas interconexiones de los diversos procesos presentes en la configuración afectiva.

\section{Palabras clave}

Afectividad, madre, padre, relación de familia.

Thesauro

Tesauro de Ciencias Sociales de la Unesco.

\section{Para citar este artículo}

Aya-Angarita, S. L., \& García-Suárez, C. I. (2020). El universo emocional en las interacciones parentofiliales: un acercamiento al estado del arte. Revista Latinoamericana de Ciencias Sociales, Niñez y Juventud, 18(1), 1-17. http://dx.doi.org/ $10.11600 / 1692715 x .18106$

\section{Historial}

Recibido: 06.05.2019

Aceptado: 24.07.2019

Publicado: 25.11.2019

Información artículo

El artículo forma parte del proyecto en curso «Configuración de prácticas y saberes afectivos en familias monoparentales de Bogotá Colombia », realizada con el apoyo de la Universidad Santo Tomás de Aquino (Usta), en las líneas Psicología, contextos cotidianos y transformaciones sociales de la Usta, e Infancias, familias y cultura. Área: Ciencias Sociales; subárea: Psicología. 


\section{The emotional universe in parent-child interactions: an approach to a literature review}

\section{Abstract (analytical)}

The present literature review paper intends to reveal the ways in which various theoretical and investigative studies have understood emotional bonding within parent-child relationships. For this purpose, and using a hermeneutical approach, 69 articles published in Ibero-America and the Caribbean and some European countries in the last 13 years were exhaustively analyzed by the authors. This helped identify that emotions in parent-child interactions are understood as control mechanisms, interwoven threads throughout life and socio-historical-cultural constructs. The findings also suggest that despite the fact that researchers use various methods and integrate different perspectives, there is no clarity achieved about the complex interconnections of various processes that are present in the affective configuration.

Keywords

Affectivity, mother, father, family relationship.

\section{O universo emocional nas interações parento-filial: uma aproximação ao estado da arte}

Resumo (analítico)

O presente artigo de revisão pretende revelar as maneiras pelas quais vários desenvolvimentos teóricos e investigativos têm entendido os mecanismos emocionais dentro das relações parento- filiais. Para este propósito, através de um exercício hermenêutico, 69 textos publicados na lbero-América e no Caribe e alguns países Europeus nos últimos 13 anos foram exaustivamente analisados. Este desenvolvimento possibilitou identificar que as emoções nas interações parento-filiais são entendidas como mecanismos de controle, como fios entrelaçados ao longo da vida e como construções sócio-histórico-culturais. As descobertas também sugerem que, apesar do fato de os pesquisadores reconhecerem vários métodos e integrarem diferentes perspectivas, não há clareza sobre as complexas interconexões dos vários processos presentes na configuração afetiva.

Palavras-chave

Afetividade, mãe, pai, relacionamento familiar.

Información autores

[*] Psicóloga y magíster en Psicología Clínica y de la Familia de la Universidad Santo Tomás. Docente de la Facultad de Psicología de la Universidad Santo Tomás. Candidata al doctorado en Ciencias Sociales, Niñez y Juventud. (iD) 0000-0002-8909-7173. Índice H5: 2. Correo electrónico: sandraaya@usantotomas.edu.co

[**] Periodista. Licenciado en Filología e Idiomas. Especialista en Comunicación-Educación. Doctor en Ciencias Sociales, Niñez y Juventud. Director de Inclusión e Integración de Poblaciones en la Secretaría de Educación de Bogotá. (iD) 0000-0002-0859-1234. Índice H5: 6. Correo electrónico: ivangarciaok@yahoo.com 


\section{Introducción}

La interacción emocional que se entreteje en la convivencia cotidiana de los seres humanos organiza las realidades que fundamentan las formas de ver el mundo (Maturana, 2002). Tal interacción empieza en las relaciones significativas, en aquellas que nos reciben en el momento en que nacemos y nos orientan frente los modos en que debemos sentir (Perinat, 2007). Con esta premisa se intuye que el engranaje emocional -el cual se da desde el momento mismo de la concepción (Cyrulnik, 2008) — se sitúa en escenarios familiares específicos que le otorgan singularidades. El propósito de este texto será, entonces, develar cómo diversos desarrollos investigativos y teóricos han interpretado este engranaje emocional, específicamente en la interacción parentofilial, con el fin de reconocer el complejo proceso que da vida al universo emocional en las relaciones más íntimas.

\section{Metodología}

La metodología implementada desde una postura hermenéutica implicó la lectura, selección, organización, clasificación e interpretación de más de 150 textos encontrados en bases de datos como Redalyc, Dialnet, Scielo, Clacso, ProQuest y EBSCO Host. En los elementos de búsqueda iniciales que orientaron los hallazgos, se combinaron tres ejes temáticos: interacción parentofilial, construcción emocional/afectiva e instauración emocional/afectiva en la vida humana. Luego de filtrar la información, se analizaron 69 manuscritos publicados entre 2005 y 2018 en Suramérica (64\%), Centroamérica (6\%), el Caribe (3\%), España (21\%) y otros países europeos (6\%). Los escritos se dividieron entre libros completos $(5 \%)$, capítulos de libros (7\%), artículos teóricos o de reflexión (17\%), manuscritos de revisión (30\%) y escritos resultados de investigación en campo (41\%). Inicialmente, los trabajos se sistematizaron en RAE (resúmenes analíticos especializados) y después la información se organizó en una matriz interpretativa, que pretendió identificar marcos disciplinares y paradigmáticos, métodos trabajados en las investigaciones, lugar 
donde se dijo lo que se dijo, objetivo al cual apuntaba el texto, resultados (en el caso de las investigaciones), elementos de desarrollo argumentativo (en el caso de los conceptos teóricos), convergencias en los discursos, divergencias y conclusiones generales.

Los criterios iniciales de inclusión para el análisis de los textos indicaron que en estos se debía abordar directa o indirectamente el tema de emociones o afectos en las interacciones de padres y madres con sus hijos e hijas; asimismo, los manuscritos se incluían si se habían escrito en Iberoamérica o el Caribe, en el periodo de tiempo señalado para el rastreo. No obstante, estos criterios se flexibilizaron por cuanto se hallaron textos que, por un lado, no estaban ubicados en los territorios inicialmente planteados y ofrecían visiones sobre las interacciones parentofiliales y, por otro, que aun cuando no hablaban en forma directa de la interacción parentofilial, sí exponían interpretaciones sobre el papel de las emociones en las interacciones humanas, todo lo cual fortaleció la comprensión conceptual del tema. Los documentos se excluían cuando, pese a que hablaran de emociones en las interacciones humanas, no aportaban a las comprensiones sobre las formas en que las emociones surgen y circulan en las relaciones íntimas de padres y madres con sus hijos e hijas.

\section{Resultados}

En el análisis se evidenció que la psicología, desde todas sus corrientes, es la disciplina que en apariencia más se interesa por este tema (59\%), seguida por la educación (18\%), la antropología y la sociología (16\%). No obstante, también se reconocieron discursos desde la psiquiatría, las neurociencias, la etología y el psicoanálisis (7\%). Asimismo, se identifica que varios de los textos posicionaron sus miradas desde posturas críticas, complejas e interpretativas (61\%) y otros tantos desde lentes tradicionales, como el positivismo (27\%). No se evidenció ninguna postura paradigmática en el $12 \%$ de los manuscritos.

En relación con las investigaciones en campo, se encontró que el 59\% de estudios fueron de tipo cualitativo, el $31 \%$ cuantitativo y el $10 \%$ mixtos. De la totalidad de estos procesos, $41 \%$ trabajaron solamente con padres, madres o cuidadoras (madres y abuelas, sobre todo), $17 \%$ realizaron el estudio con niños, niñas y adolescentes mayores de siete años y $34 \%$ llevaron a cabo la investigación con toda la familia. Frente a este último, vale la pena aclarar que solo un estudio integró en un mismo escenario a todos los miembros de la familia; los otros aplicaron las técnicas investigativas por separado. 
La revisión de todos los textos ayudó además a identificar tres tendencias frente a las maneras en que las emociones son comprendidas en las interacciones parentofiliales. La primera de ellas sugiere que las emociones son elementos o dispositivos de control hacia la infancia; la segunda alude a que las emociones son hilos que tejen experiencias a lo largo de la vida; mientras que la tercera plantea que las emociones son constructos sociales en los que se involucran escenarios sociales, históricos y culturales.

En las tres tendencias, el discurso que se ha empoderado del tema es la psicología; además, se evidencia que sobre todo la psicología cognitivo-comportamental hace importantes aportes, seguida de las psicologías construccionista, discursiva y sistémica. Asimismo, se identifica que son numerosos los estudios y comprensiones que trabajan desde métodos cualitativos y desde posturas relacionales e interpretativas. Sin embargo, pese a que se identifican multiplicidad de saberes, se percibe que estos exponen sus comprensiones de manera fragmentada y no establecen claridad sobre las complejas interconexiones de los procesos presentes en la configuración afectiva. Se nota, además, que todos los estudios ponen énfasis en la responsabilidad de los adultos significativos (especialmente en las madres) en la vida de niños, niñas y adolescentes para un desarrollo emocional saludable, e identifican una feminización en la transmisión y el cuidado afectivo.

\section{Las emociones como elementos de control}

En el marco de estas interpretaciones, las emociones son dispositivos de control usados por el mundo adulto para favorecer la adaptación de sus hijos e hijas a las demandas del contexto. Se interpreta desde estas visiones que las emociones y sus formas de significación y manifestación son responsabilidad de los individuos que las poseen. Tales perspectivas se han desarrollado en su mayoría por medio de discursos desde la fisiología, la genética, las teorías evolucionistas y la psicología cognitivo-conductual.

En estas posiciones se encuentran estudios iniciales desde enfoques naturalistas que basan sus observaciones en elementos fisiológicos, biológicos y genéticos, interesándose por los movimientos del cuerpo y las expresiones faciales; argumentan que es allí donde es posible observar y entender el surgimiento de las emociones (Carrascal, 2016). Tales visiones las sitúan en los desarrollos internos de los seres humanos y explican que se definen por los cambios corporales manifestados tras la aparición de un objeto significativo; es decir, son causadas por las percepciones del sujeto sobre la transformación de su propio cuerpo (William James, citado por Hernández, 2009; Melamed, 2016). 
Por su parte, las teorías evolucionistas fundamentadas en el trabajo de Charles Darwin se interesan principalmente en la socialización de las emociones a través de la conducta, definiendo a las emociones como un medio de supervivencia y como un producto de muchas adaptaciones evolutivas. En esta línea, pero haciendo una integración con las teorías naturalistas, Antonio Damasio identifica que los componentes fisiológicos y evolutivos son parte de su aparición (Hernández, 2009).

Las teorías cognitivas también han tenido gran relevancia en la comprensión y estudio de las emociones. Esta corriente, que incluye a autores como Robert Solomon (Carrascal, 2016; Gil, 2016; Melamed, 2016), las entiende como mecanismos voluntarios que involucran juicios integrados por valores e ideales sobre nosotros mismos y sobre nuestro lugar en el mundo; en resumen, organizan lo que pensamos y las experiencias de realidad que tenemos (Melamed, 2016).

Especificamente, en las relaciones parentofiliales se identifica que, siguiendo esta última línea de trabajo, la psicología cognitivo-conductual exalta la responsabilidad de los adultos para un desarrollo emocional saludable de jóvenes, niños y niñas. Los estudios indican, además, que según las formas en que padres y madres formen o moldeen las emociones de sus hijos e hijas, fomentarán su desarrollo emocional y, además, establecerán un perfil psicológico determinado.

Ejemplo de lo anterior lo ofrece el concepto de regulación emocional (Aragón et al., 2009; Colette et al., 2017; Estévez \& Jiménez, 2017; Jaramillo et al., 2017; Martínez \& García, 2011; Pérez \& Guerra, 2014; Rendón, 2007), el cual asume a las emociones como eje central para una vida sana; estas, según lo encontrado, las deben modular, controlar, redireccionar, modificar, ajustar, manejar o influenciar los adultos a cargo de niños, niñas y adolescentes, con el fin de ayudarles a adaptarse a los contextos sociales. Cuando se tiene éxito en esta labor, los estudios sugieren que se introyectan habilidades o competencias sociales, como consecución rápida de metas, relaciones saludables, estabilidad en los compromisos asumidos, formas de expresión emocional asertiva vinculadas a altos niveles de inteligencia emocional y, en general, bienestar a lo largo de la vida. Cuando se fracasa en la tarea, se generan situaciones como el consumo de sustancias psicoactivas, los trastornos de ansiedad y de pánico, los problemas de salud y los bajos niveles de inteligencia emocional.

Otros ejes conceptuales que involucran estas formas de entender las emociones son los estilos parentales (Fuentes et al., 2015; Henao \& García, 2009; Ramírez-Lucas et al., 2015; Ruvalcaba et al., 2012; Tascón, 2010;) las pautas de crianza (Castillo \& Merino, 2018; 
Cuervo, 2010; Izzedin \& Pachajoa, 2009; Puentes \& Arboleda, 2017) y algunas interpretaciones sobre los vínculos afectivos (Pérez \& Arrázola, 2013). Estas miradas, cuyas bases conceptuales en su mayoría también las ha desarrollado la psicología cognitivo-conductual, se interesan por hacer correlaciones entre dichos estilos o prácticas y sus consecuencias en la vida de niños, niñas y adolescentes.

En tales investigaciones se reconoce la presencia de las emociones ligadas al amor como trascendentes para los acompañamientos sanos al mundo infantil que hacen los adultos. Estilos democráticos o equilibrados traen consigo el respeto de normas por parte de jóvenes, niñas y niños, un ajuste psicológico adecuado y el desarrollo de la inteligencia emocional. Por el contrario, estilos autoritarios, permisivos o no implicados revierten en la infancia y en la adolescencia con diversos problemas socioafectivos. Tales estilos incluyen prácticas como castigos violentos, control excesivo, manipulación emocional, retirada del afecto y baja o nula supervisión.

\section{Las emociones como hilos que tejen experiencias a lo largo de la vida}

En este eje conceptual, también trabajado sobre todo por la psicología, las emociones se comprenden como hilos que se entretejen de formas interdependientes e inesperadas para crear las experiencias emocionales a lo largo de la vida. Tales hilos se tejen en la relación primaria (madre en especial, padre o cuidador significativo) y se proyectan a lo largo de la historia de los sujetos y sus diferentes ámbitos. A diferencia de la tendencia anterior, estas interpretaciones muestran de modo incipiente una interacción entre el mundo adulto y la infancia y la adolescencia, lo que lleva a pensar que las emociones son relacionales. El análisis de los textos indicó que en estas perspectivas se incluyen categorías como vínculos afectivos, competencias parentales y algunas visiones sobre la crianza.

El psicoanálisis (Lutenberg, 2014), la psicología (Hernández, 2010; Serebrinsky, 2009), la psiquiatría y la etología (Bowlby, 2014; Cyrulnik, 2008) han trabajado el tema de vínculos afectivos. Estas comprensiones se han interesado sobre todo en la díada madre-hijo(a) y han identificado que en dichas interacciones deben existir «bases seguras»o entornos de confianza que incluyan lazos afectivos representados por un amor que legitima al otro desde el respeto y que ayude a desplegar su autonomía a lo largo de su vida.

En consonancia con lo anterior, todas las investigaciones rastreadas (Gómez, 2015; Pineda, 2013; Rossetti-Ferreira \& Costa, 2012; Sánchez, 2011; Sarmiento et al., 2009; Tello- 
Casany et al., 2009; Villavicencio \& Villarroel, 2017) destacan la importancia de vínculos «adecuados, equilibrados o sanos» en la infancia, caracterizados por la presencia de padres y madres (sobre todo madres) sensibles a las necesidades emocionales de niños, niñas y adolescentes, lo cual se representa en su seguridad y libertad; en otras palabras, en una vida feliz.

Por otro lado, en estos estudios se identifica que las interacciones «insanas» se caracterizan por adultos significativos ausentes o intermitentes, autoritarios, violentos o despreocupados, que imponen normas muy rígidas o muy laxas y que no expresan emociones ligadas al amor empático. Además, se presentan entornos que generan inseguridad (traslado frecuente de vivienda o ambivalencia emocional por parte de los padres) y presencia sostenida de emociones en ellos y en los adultos, como el miedo, la rabia, la frustración y la tristeza. Tales interacciones llevan a instaurar en niños, niñas y adolescentes formas de actuar que transgreden las normas sociales establecidas y repercuten en su historia de vida.

Otros elementos conceptuales que se desarrollan bajo esta línea de trabajo son las competencias parentales (Ossa et al., 2018; Ramos, 2017) y la crianza en el desarrollo emocional (Oudhof et al., 2012; Pi \& Cobián, 2009; Vilches, 2007). Se identifica que especialmente la psicología y algunas interpretaciones desde la educación apoyan las comprensiones del tema.

En general, se observa que estas perspectivas reconocen interdependencia emocional en la interacción parentofilial, enfocando las miradas en padres y madres (sobre todo madres) para el desarrollo sano de la infancia y la adolescencia. Además, las investigaciones identificadas alrededor de este tema, por un lado, reconocen formas de interacción de padres, madres con sus hijos e hijas en contextos de vulnerabilidad y, por otro, invitan a estos padres y madres a formarse para acompañar el desarrollo emocional de sus hijos e hijas, así como a introyectar las competencias parentales necesarias para potenciar los recursos presentes en la vida de niños, niñas y adolescentes, ofreciéndoles mejores posibilidades de vida. Dichas competencias se refieren a empatía para la atención oportuna de las necesidades tanto físicas como emocionales de jóvenes, niños y niñas, flexibilidad y sensibilidad frente a las transformaciones de todas sus dimensiones, posibilidad de establecer redes de apoyo ante el cuidado y la crianza, facilidad de adaptarse frente a las demandas de los contextos, creatividad para usar los recursos disponibles y habilidad comunicativa para expresar de modo asertivo las emociones, entre otros aspectos. 


\section{Las emociones como constructos sociohistoricoculturales}

Con estas miradas, cuyos discursos se enmarcan en la psicología (sistémica, compleja y discursiva), la antropología, la sociología y la educación, se reconoce que las emociones no solo surgen y se conectan en la subjetividad individual y en la intersubjetividad familiar, sino que además se entremezclan en un complejo entramado social, histórico y cultural (Barudy \& Dantagnan, 2007; Hernández, 2010; Perinat, 2007). Todo lo cual deriva en reglas de interacción que circulan a través de la comunicación (símbolos, códigos, palabras, expresiones físicas, etc.) y crea realidades colectivas sobre las formas de emocionar (Chávez, 2013; Enciso \& Lara, 2014; Flórez, 2017; Florido, 2016; Gallego, 2012; Le Breton, 2012; López, 2017).

Esta comunicación se transmite por medio de actos discursivos (Belli et al., 2010; Belli \& Íñiguez, 2008; Enciso \& Lara, 2014) en el intercambio social cotidiano (Bericat, 2012) y se recrea a través de prácticas patriarcalistas (Sánchez \& Palacios, 2013; Varela et al., 2015) que otorgan significados a las emociones ligadas al cuidado y la crianza, según el género (García et al., 2010; Mora, 2005), feminizando así esta labor (Botero, 2017; García, 2017; López, 2012; Moreno et al., 2018; Varela et al., 2015) e instituyen inequidades. De otra parte, tales prácticas legitiman la violencia y el autoritarismo como forma de control emocional (Bedoya, 2013; Flórez, 2017), otorgando a la masculinidad -y, por ende, a la paternidad-el poder de control sobre aquello que en apariencia es más débil (Quintero \& Thevenot, 2015); ello se manifiesta en el castigo físico y la «retirada del afecto» o en la manipulación emocional (Bocanegra, 2007).

Lo anterior probablemente se instauró desde la colonización de las Américas (Bocanegra, 2007) y se legitimó a lo largo de los años (Varela et al., 2015), dada la invisibilización de discursos locales (Chávez, 2013) y la naturalización de interpretaciones científicas impuestas por instituciones reguladoras; estas establecen estilos de cuidado generalizados y estandarizados, al igual que modelos únicos de parentalidad (Álvarez, 2016) y, por consiguiente, de afectividad.

En relación con los contextos sociales, se identifica que las emociones no solo emergen y se manifiestan en la díada madre/padre-hijo(a); en su significación entran en juego otras redes de apoyo o constelaciones de cuidado, compuestas por abuelos y abuelas, comunidades circundantes a la familia (vecinos y amigos) y algunas instituciones, como los jardines infantiles o escuelas (Bedoya, 2013; Flórez, 2017; Hernández, 2017; Marín \& Palacios, 2015; Moreno, 2012; Rossetti-Ferreira \& Amaral, 2012), creando así entornos afectivos seguros para niños, niñas y adolescentes (López, 2012). 
Según las investigaciones rastreadas, esta construcción emocional se enmarca en escenarios de interacción social con condiciones políticas y económicas específicas, como la pobreza (García, 2017; Moreno, 2012), la ausencia física y afectiva de madres y padres por mantener un puesto de trabajo (Barudy \& Dantagnan, 2007; Marín \& Palacios, 2015; Marín \& Palacios, 2016; Varela et al., 2015), la migración obligada de alguno de los padres (López, 2012) y la confrontación cultural por raza y etnia (Chávez, 2013).

Por último, en relación con esta tendencia se identifica que la interacción emocional de padres y madres con sus hijos e hijas está atravesada por las transiciones ideológicas y sus consecuentes cambios estructurales en el interior de la familia (García, 2017; Llanos, 2017; Montagna, 2016; Moreno, 2013; Palacios, 2009). Estas se caracterizan por formas alternas de expresión afectiva, que dan cabida al amor compasivo, comprensivo y libre de violencia (Llanos, 2017); no obstante, pese a las nuevas formas de interacción, se reconocen tensiones y, con ellas, prácticas que aún se anclan en las concepciones tradicionales (Llanos, 2017; Palacios, 2009).

\section{Discusión}

Además de cumplir con el objetivo principal del artículo, el cual buscaba evidenciar las maneras en que diversos desarrollos teóricos e investigativos comprenden la interacción afectiva en las relaciones parentofiliales, se observa como conclusión general que, pese a que hay tres tendencias que abarcan tales comprensiones (las emociones como mecanismos de control, las emociones como hilos que tejen experiencias a lo largo de la vida y las emociones como constructos sociohistoricoculturales), los discursos no evidencian las complejas interconexiones de los procesos presentes en la configuración afectiva.

Esto se podría sustentar en el hecho de que la mayoría de los trabajos se instauran desde visiones discursivas que no establecen diálogos con otras disciplinas. Asimismo, podría reconocerse en el hecho de que si bien se trabaja desde variados métodos asumidos con diversas perspectivas, los resultados suelen presentar solo una parte que integra el todo emocional, desconociendo la interdependencia y la recursividad que existen entre cada mecanismo presente en el entramado afectivo.

En cuanto a las interpretaciones generales, en la mayoría de los estudios se magnifica la relación entre el mundo femenino y el emocional, desconociendo en muchos casos la voz de las masculinidades en las interacciones afectivas. Además, muchas investiga- 
ciones centran sus propósitos en comprender las acciones de los adultos sobre el desarrollo socioafectivo de niños, niñas y adolescentes, desestimando los propios procesos afectivos de padres y madres, al igual que la importancia que tiene la participación de la infancia y la adolescencia en la construcción emocional de los primeros.

En consonancia con lo anterior, los hallazgos llevan a interpretar que la interacción afectiva parentofilial en territorios iberoamericanos está bajo el dominio femenino, lo que se ve representado en la feminización del cuidado y de la crianza o en la gran relevancia otorgada a la diada madre-hijo(a). Frente a esto se identifica que existen fuertes desequilibrios que cargan a las mujeres, quienes, a pesar de sus esfuerzos, en algunos casos se dejan ir tras la inercia de una cultura que ha naturalizado y legitimado la violencia en el marco del capitalismo y el patriarcalismo. No obstante, también se identifica que, sin importar las circunstancias, las mujeres cuentan con sinnúmero de recursos emocionales que les permiten transformar las realidades de sus hijos e hijas y establecer, entre otras cosas, ambientes seguros, instaurando alrededor de niños, niñas y adolescentes constelaciones de cuidado.

Los hallazgos también muestran que son múltiples las investigaciones que confirman que los entornos afectivos y seguros traen consigo grandes beneficios para el desarrollo y el bienestar social y emocional de niños, niñas y adolescentes. También se reconoce en varios estudios que los entornos cuyas prácticas de interacción están mediadas por privación del afecto repercuten en situaciones conflictivas. Pese a lo evidente, al parecer algunos ambientes en Latinoamérica están mediados más por la segunda dinámica que por la primera, como reflejo de historias de violencia y exclusión que ubican el miedo y la ira en la interacción afectiva entre padres e hijos y que manifiestan estilos autoritarios que deslegitiman las emociones como parte de la naturaleza humana.

Se comprende entonces que las emociones son cimientos de la vida humana (Maturana, 2002) y se configuran en un complejo entramado entre los ámbitos subjetivo (cognición, biología, experiencias particulares) y contextual (significados, creencias, ideologías e historia), en el que se producen intersubjetividades a través de símbolos culturales que transitan por medio del lenguaje; así, estas definen las historias humanas y, al mismo tiempo, son definidas y transformadas por la historia humana.

En congruencia con lo expuesto, se reconoce que para ampliar las miradas sobre este tema será importante desarrollar dos frentes de acción. El primero se relaciona con los escenarios cotidianos de las familias, en cuanto se evidencia que en las intimidades parentofiliales, ideologías hegemónicas como el patriarcalismo y el capitalismo dominan 
los afectos y sus múltiples formas de expresión, negando, entre otras cosas, la presencia de emociones ligadas al cuidado y al amor compasivo y de masculinidades sensibles, y sobredimensionando la responsabilidad de las feminidades. En este frente habrá que trabajar con las cotidianidades familiares y sus significados de ser familia, integrando a las emociones como parte fundamental en su desarrollo y evolución de todos los integrantes.

El segundo frente corresponde al ámbito académico, el cual, además de ampliar sus fronteras discursivas para entender el fenómeno de manera transdisciplinar, debe propiciar diálogos generativos entre varios saberes (entre ellos los científicos y los populares); incluiría así diferentes voces y versiones de quienes viven la experiencia afectiva, para comprender otras maneras de engranaje emocional, entendiendo que las posibles limitaciones de un estudio de este tipo radicarían en la poca flexibilidad de los discursos dominantes.

\section{Referencias}

Álvarez, C. (2016). Crianza-regulación, crianza-emancipación: estado de la cuestión de estudios sobre la crianza. Aletheia, 8(1), 80-99. https://doi.org/ 10.11600/21450366.8.1aletheia.80.99

Aragón, R., Cruz, L., \& Carrasco, E. (2009). Configuración semántica de la RE, control, enfrentamiento e inteligencia emocional. Revista Electrónica de Motivación y Emoción, 11(31). http://reme.uji.es/articulos/numero31/article4/texto.html

Barudy, J., \& Dantagnan, M. (2007). Los buenos tratos de la infancia: parentalidad, apego y resiliencia. Gedisa.

Bedoya, M. H. (2013). Redes del cuidado: ética del destino compartido en las madres comunitarias antioqueñas. Revista Latinoamericana de Ciencias Sociales, Niñez y Juventud, 11(2), 741-753. https://doi.org/10.1160o/1692715x.11219140812

Belli, S., Harré, R., \& Íñiguez, L. (2010). Emociones y discursos: una mirada a la narrativa científica de la construcción social del amor. Prisma Social, (4), 1-45.

Belli, S., \& Íñiguez, L. (2008). El estudio psicosocial de las emociones: una revisión y discusión de la investigación actual. Psico, 39(2), 139-151.

Bericat, E. (2012). Emociones. Sociopedia.isa, 1-13.

Bocanegra, E. M. (2007). Las prácticas de crianza entre la Colonia y la independencia de Colombia: los discursos que las enuncian y las hacen visibles. Revista Latinoamericana de Ciencias Sociales, Niñez y Juventud, 5(1), 201-231. 
Botero, A. (2017). De las violencias al buen trato y prácticas familiares en la crianza [Tesis de maestría, Universidad de Manizales]. Repositorio Institucional Universidad de Manizales. http://ridum.umanizales.edu.co:8080/xmlui/handle/6789/3011

Bowlby, J. (2014). Vínculos afectivos: formación, desarrollo y pérdida. Morata.

Carrascal, L. F. L. (2016). Las emociones como formas de implicación en el mundo. El caso de la ira. Estudios de Filosofía, (53), 81-101.

Castillo, S., \& Merino, Z. (2018). La desintegración familiar: impacto en el desarrollo emocional de los niños. Revista Ciencia e Investigación, 3(9), 10-18. https://doi.org/ 10.26910/issn.2528-8083volziss9.2018pp10-18p

Chávez, M. (2013). La familia, las relaciones afectivas y la identidad étnica entre indígenas migrantes urbanos en San Luis Potosí. Estudios de Historia y Sociedad, XXXIV (134), 131-155.

Colette, S., Restrepo, D., Moreno, M., Hoyos, O., \& Palacio, J. (2017). Emotion regulation in children and adolescents: Concepts, processes and influences. Psicología desde el Caribe, 34(1). https://doi.org/10.14482/psdc.34.1.9778

Cuervo, Á. (2010). Pautas de crianza y desarrollo socioafectivo en la infancia. Revista Diversitas, 6(1), 111-121. https://doi.org/10.15332/s1794-9998.2010.0001.08

Cyrulnik, B. (2008). Bajo el signo del vínculo: una historia natural de apego. Gedisa.

Enciso, G., \& Lara, A. (2014). Emociones y ciencias sociales en el s. XX: la precuela del giro afectivo. Athenea Digital. Revista de Pensamiento e Investigación Social, 14(1), 263-288. https://doi.org/10.5565/rev/athenead/v14n1.1094

Estévez, E., \& Jiménez, T. (2017). Violencia en adolescentes y regulación emocional. International Journal of Developmental and Educational Psychology, 2(1), 97-104. https:// doi.org/10.17060/ijodaep.2017.n1.v2.922

Flórez, G. L. (2017). Los tejidos de la crianza: vínculos y capacidades de los sujetos que cuidan [Tesis de maestría, Universidad de Manizales]. Repositorio Institucional Universidad de Manizales. http://ridum.umanizales.edu.co:8080/xmlui/handle/6789/3011

Florido, L. (2016). El estudio sociohistórico de las emociones y los sentimientos en las ciencias sociales del siglo XX. Revista de Estudios Sociales, (55), 178-191. https:// doi.org/10.7440/res55.2016.12

Fuentes, M., García, F., Gracia, E., \& Alarcón, A. (2015). Los estilos parentales de socialización y el ajuste psicológico: un estudio con adolescentes españoles. Revista de Psicodidáctica, 20(1), 117-138. https://doi.org/10.1387/RevPsicodidact.10876

Gallego, T. (2012). Familias, infancias y crianza: tejiendo humanidad. Revista Virtual Universidad Católica del Norte, (35), 63-82. 
García, E., Salguero, A., \& Pérez, G. (2010). Expectativas y estereotipos de género en la relación entre padres e hijas. Enseñanza e Investigación en Psicología, 15 (2), 325-341.

García, L. (2017). Habilidades para la crianza en las figuras paterna y materna [Tesis de maestría, Universidad de Manizales]. Repositorio Institucional Universidad de Manizales. http://ridum.umanizales.edu.co:8080/xmlui/handle/6789/3011

Gil, M. (2016). La complejidad de la experiencia emocional humana: emoción animal, biología y cultura en la teoría de las emociones de Martha Nussbaum. Dilemata, 8(21), 207-225. Recuperado de https://www.dilemata.net/revista/index.php/ dilemata/article/view/412000020

Gómez, M. J. (2015). La crianza natural como modelo emergente de educación emocional en la familia: análisis de prácticas de crianza y de conversaciones de mensajería instantánea en una comunidad virtual de apoyo [Tesis doctoral no publicada]. Universidad de Las Palmas de Gran Canaria.

Henao, G., \& García, M. (2009). Interacción familiar y desarrollo emocional en niños y niñas. Revista Latinoamericana de Ciencias Sociales, Niñez y Juventud, 7(2), 785-802.

Hernández, A. (2010). Vínculos, individuación y ecología humana: hacia una psicología clínica compleja. Editorial Universidad Santo Tomás.

Hernández, D. (2009). Una perspectiva de las teorías de la emoción: hacia un estudio de las implicaciones de las emociones en la vida del hombre [Tesis de pregrado, Universidad Colegio Mayor de Nuestra Señora del Rosario]. Repositorio Institucional E-docUR, Universidad del Rosario. http://repository.urosario.edu.co/handle/10336/1772

Hernández, N. E. (2017). Redes de apoyo en la crianza [Tesis de maestría, Universidad de Manizales]. Repositorio Institucional Universidad de Manizales. http:// ridum.umanizales.edu.co:8080/xmlui/handle/6789/3011

Izzedin, B., \& Pachajoa, L. A. (2009). Pautas, prácticas y creencias acerca de crianza: ayer y hoy. Liberabit. Revista de Psicología, 15(2), 109-115.

Jaramillo, J. M., Rendón, M. I., Muñoz, L., Weis, M., \& Trommsdorff, G. (2017). Children's self-regulation in cultural contexts: The role of parental socialization theories, goals, and practices. Frontiers in Psychology, 8(923). https://doi.org/10.3389/ fpsyg.2017.00923

Le Breton, D. (2012). Por una antropología de las emociones. Revista Latinoamericana de Estudios sobre Cuerpos, Emociones y Sociedad, 4(10), 67-77.

Llanos, N. (2017). No quiero lo mismo para mis hijos: cambios y permanencias en el ejercicio de la crianza. [Tesis de maestría, Universidad de Manizales]. Repositorio Institucional Universidad de Manizales. http://ridum.umanizales.edu.co:8080/xmlui/handle/6789/3011 
López, L. (2012). El cuidado de las hijas y los hijos durante la migración internacional de los padres y las madres. Ánfora, 19(32), 117-136. https://doi.org/10.30854/ anf.v19.n32.2012.75

López, L. E. (2017). El juego y su papel vinculante en la crianza para la paz. [Tesis de maestría, Universidad de Manizales]. Repositorio Institucional Universidad de Manizales. http://ridum.umanizales.edu.co:8080/xmlui/handle/6789/3011

Lutenberg, J. (2014). Teoría de los vínculos en psicoanálisis. Cauces Editores.

Marín, A., \& Palacio, M. C. (2015). El abuelazgo: enlace intergeneracional en la crianza y cuidado de la primera infancia. Revista Latinoamericana de Estudios de Familia, 7, 11-27.

Marín, A., \& Palacio, M. C. (2016). La crianza y el cuidado en primera infancia: un escenario familiar de inclusión de los abuelos y las abuelas. Trabajo Social, 18, 159-176.

Martínez, M., \& García, M. C. (2011). Implicaciones de la crianza en la regulación del estrés. Revista Latinoamericana de Ciencias Sociales, Niñez y Juventud, 9(2), 535-545.

Maturana, H. (2002). Transformación en la convivencia. Dolmen.

Melamed, A. (2016). Las teorías de las emociones y su relación con la cognición: un análisis desde la filosofía de la mente. Cuadernos de la Facultad de Humanidades y Ciencias Sociales-Universidad Nacional de Jujuy, (49), 13-38.

Montagna, P. (2016). Parentalidad socioafectiva y las familias actuales. Derecho PCP, (77), 219-233. https://doi.org/10.1880o/derechopucp.201602.010

Mora, M. (2005). Emoción, género y vida cotidiana: apuntes para una intersección antropológica de la paternidad. Espiral, $12(34), 09-35$.

Moreno, M. (2012). Interacciones vinculares en el sistema de cuidado infantil en contextos de pobreza y desnutrición crónica temprana. [Tesis doctoral no publicada]. Universidad de Manizales-Cinde.

Moreno, M., Agudelo, M., \& Alzate, V. (2018). Voces a escuchar en el cuidado: ¿qué dicen los niños y las niñas? Revista Latinoamericana de Ciencias Sociales, Niñez y Juventud, 16(1), 227-237. https://doi.org/10.1160o/1692715x.16113

Moreno, N. (2013). Familias cambiantes, paternidad en crisis. Psicología desde el Caribe, 30(1), 177-209.

Ossa, D., Pérez, D., \& Prado, A. (2018). Competencias parentales y resiliencia infantil en contexto de desplazamiento en Colombia. Prisma Social, 20(1), 227-253.

Oudhof, H., Rodríguez, B., \& Robles-Estrada, É. (2012). La percepción de la crianza en padres, madres e hijos adolescentes pertenecientes al mismo núcleo familiar. Liberabit. Revista de Psicología, 18(1), 75-81. 
Palacios, M. C. (2009). Los cambios y transformaciones en la familia: una paradoja entre lo sólido y lo líquido. Revista Latinoamericana de Estudios Familiares, (1), 46-60.

Pérez, B., \& Arrázola, E. (2013). Vínculo afectivo en la relación parentofilial como factor de calidad de vida. Tendencias y Retos, 8(1), 17-32.

Pérez, Y., \& Guerra, V. (2014). Emotion regulation and its implications for the adolescent's health. Revista Cubana de Pediatría, 86(3), 368-375.

Perinat, A. (2007). Psicología del desarrollo: un enfoque sistémico. Universitat Oberta de Catalunya.

Pi, A., \& Cobián, A. (2009). Componentes de la función afectiva familiar: una nueva visión de sus dimensiones e interrelaciones. Medisan, 13(6).

Pineda, A. (2013). Los vínculos afectivos en las familias como recurso ante la vulnerabilidad. Aletheia, 5(2). 90-107. https://doi.org/10.11600/21450366.5.2aletheia.90.107

Puentes, A., \& Arboleda, M. (2017). Prácticas de crianza y trastornos psicológicos en adolescentes colombianos. Psicología Conductual, 25(3), 599-621.

Quintero, J., \& Thevenot, A. (2015). Imaginario social del padre, imago paterno y función paterna: reflexiones y preguntas sobre el maltrato infantil y la autoridad parental. Informes Psicológicos, 15(2), 139-163. https://doi.org/10.18566/infpsicv15n2ao8

Ramírez-Lucas, A., Ferrando, M., \& Sainz, A. (2015). ¿Influyen los estilos parentales y la inteligencia emocional de los padres en el desarrollo emocional de sus hijos escolarizados en $2^{\underline{0}}$ ciclo de educación infantil? Acción Psicológica, 12(1), 65-78. https:// doi.org/10.5944/ap.12.1.14314

Ramos, K. (2017). Aptitudes parentales de las familias en condición de pobreza y desplazamiento forzado. [Tesis de maestría, Universidad de Manizales]. Repositorio Institucional Universidad de Manizales. http://ridum.umanizales.edu.co:8080/xmlui/ handle/6789/3011

Rendón, M. I. (2007). Regulación emocional y competencia social en la infancia. Diversitas, 3(2), 349-363. https://doi.org/10.15332/s1794-9998.2007.0002.13

Rossetti-Ferreira, M., \& Amaral, N. (2012). Construcción de vínculos afectivos en contextos adversos de desarrollo: importancia y polémicas. Revista Scripta Nova. Revista Electrónica de Geografía y Ciencias Sociales, XVI(395).

Ruvalcaba, N., Gallegos, J., Robles, F., Morales, A., \& González-Gallego, N. (2012). Inteligencia emocional en la mejora de los estilos educativos de padres con hijos en edad escolar. Salud \& Sociedad, 3(3), 283-291.

Sánchez, G., \& Palacio, M. (2013). Cuidado familiar, orden discursivo hegemónico y contra-hegemónico. Revista Latinoamericana de Estudios de Familia, 5, 29-45. 
Sánchez, P. (2011). La experiencia vinculante afectiva del sujeto adolescente infractor. Revista Latinoamericana de Ciencias Sociales, Niñez y Juventud, 10(1), 453-465.

Sarmiento, A., Puhl, S., Oteyza, G., Bermúdez, F., \& Siderakis, M. (2oog). Las conductas transgresoras de los adolescentes en conflicto con la ley penal; su relación con las perturbaciones en el vínculo primario. Anuario de Investigaciones, XVI, 51-57.

Serebrinsky, H. (2009). Un viaje circular: de la psicología social pichoniana a la teoría sistémica. Editorial de los Cuatro Vientos.

Tascón, M. (2010). Influencia de los estilos parentales en la construcción de la norma de niños y niñas de cinco a seis años del Colegio mixto Juan Pestalozzi de la ciudad de Cali [Tesis de maestría no publicada]. Universidad de Manizales-Cinde.

Tello-Casany, C., Mazur, A., \& Vidal-Bota, J. (2009). Vinculación afectiva prenatal. Cuadernos de Bioética, XX(3), 548-550.

Varela, S., Chinchilla, T., \& Murad, V. (2015). Prácticas de crianza en niños y niñas menores de seis años en Colombia. Zona Próxima, (22), 193-215. https://doi.org/ 10.14482/zp.22.6129

Vilches, L. (2007). Análisis de aspectos emocionales en las interacciones familiares y en la educación de los hijos. Educación y Futuro, (17), 105-130.

Villavicencio, C., \& Villarroel, M. (2017). Comunicación afectiva en familias desligadas. Revista de difusión cultural y cientifica de la Universidad La Salle en Bolivia, 13(13), 15-39. 